\title{
Overcoming Obesogenic Environments: A Qualitative Study Exploring Mechanisms of Healthy Eaters
}

\author{
Sofía Sutherland Borja ${ }^{1}$, Emilio A González Silva ${ }^{2,4 *}$, Cinthya Urquidi \\ Bellota $^{3}$, Gabriela Sarrat Gonzalez ${ }^{1}$ and Eliana Reyes Soto ${ }^{1}$ \\ ${ }^{1}$ School of Nutrition and Dietetics, Faculty of Medicine, Universidad de los Andes, \\ Chile \\ ${ }^{2}$ Physical Medicine and Rehabilitation Service, Hospital de Urgencia de la Asisten- \\ cia Pública (HUAP), Santiago, Chile \\ ${ }^{3}$ Department of Public Health, Faculty of Medicine, Universidad de los Andes, Chile \\ ${ }^{4}$ Universidad del Desarrollo, Facultad de Medicina Clínica Alemana, Chile \\ *Corresponding Author: Emilio A González Silva, Physical Medicine and Rehabilita- \\ tion Service, Hospital de Urgencia de la Asistencia Pública (HUAP), Santiago, Chile. \\ E-mail: emiliogonzalez.kine@gmail.com
}

Received: February 05, 2021

Published: February 26, 2021

(C) All rights are reserved by Sofía Sutherland Borja., et al.

\begin{abstract}
Objective: To identify mechanisms that enable overcoming obesogenic environments amongst healthy eaters through a Salutogenesis approach.

Methods: Sixteen middle-class Chilean adults (14 females and 2 males) following healthy diets according to the Healthy Eating Index were purposively selected from a primary health care center in urban Santiago. Semi-structured interviews and a grounded theory approach were used to explore ways in which healthy eaters manage their food practices. Interview transcripts were coded through open, axial and selective coding with a constant comparative method.

Results: The link between food and health is the main mechanism that promotes healthy diets. Healthy eaters assign importance to the physical and emotional roles of food, and use knowledge, motivation and resource-management skills to manage healthy food practices. A three stage food-related learning processes is proposed.

Conclusion: Understanding healthy eating as fundamental to reach a state of well-being and having skills to perform adequate food practices is key for facing obesogenic environments. The health-food link and the Food Learning Triad may serve for nutrition education interventions.
\end{abstract}

Keywords: Salutogenesis; Eating Behavior; Healthy Diet; Health Promotion; Food Coping Mechanisms

\section{Introduction}

During the last decades, the nutritional transition occurred in Chile has generated an obesogenic environment, in which sedentary lifestyle, availability and access to highly processed foods, and low consumption of nutritious ones have seriously impacted eating habits, nutrition and health of the Chilean population [1,2]. According to the National Food Consumption Survey from 2010, only $5 \%$ of Chileans report following a healthy diet, while the remaining $95 \%$ require significant change in their eating habits. In turn, the survey reflects noncompliance of food guidelines, particularly of fruits, vegetables and fish; in addition to excessive consumption of calories, saturated fats, sugars and sodium [3]. The consequence of such eating habits has resulted in high prevalence of excess body weight and chronic non-communicable diseases as diabetes and hypertension [4].

Studies aimed at dietary behaviors and nutritional status have been framed within the pathogenesis scope, i.e. by analyzing the origin of pathologies to treat and prevent diseases, providing extensive knowledge about risk factors associated with excess malnutrition, such as educational level, income level, availability and 
access to food, nutritional knowledge, etc [5]. Consequently, health promotion and nutrition strategies have focused mainly on risk factors $[2,6,7]$. Despite these efforts, national surveys do not reflect a reduction of the problem [1,2], suggesting the need to address the situation from a different angle.

Salutogenesis is a theoretical perspective on the development of health proposed by Aaron Antonovsky in the 70's. Contrasting to 'pathogenesis', it studies the origins of health, and explains health as a dynamic state in which individuals move along a continuum, between extremes of absolute health ('ease' end of the continuum) and lack thereof ('dis-ease' end of the continuum). One's position in the ease/disease continuum depends on the ability to identify and use resources at one's disposal that allow managing the various situations of daily life, which determine the state of more or less health; ability conceptualized as Sense of Coherence (SOC) [8-11]. Salutogenesis applied to the context of nutrition provides a lens for studying factors that favor healthy dietary behaviors, paying attention to those people who are able to maintain a healthy diet despite the obesogenic environment.

Healthy eating habits in adults are positively associated to social and psychological factors such as cohabitation, positive health perception, ability to handle adverse situations, flexible dietary restriction and self-efficacy in relation to food [12], as well as positive values and beliefs related to health and nutrition, concern for environmental sustainability and having food skills (e.g. handling time and money for proper food selection and basic cooking skills) [13]. On the other hand, factors such as high educational level and income, knowledge of nutrition and social support were not associated with better eating habits [12]. Furthermore, a strong SOC is associated to healthier behaviors related to food and eating [1420].

Health promotion aims for people being in control over their health and improve it. Hence, there is a need to further explore the different elements that facilitate the adoption of healthier dietary behaviors in obesogenic food environments to better address malnutrition and its associated diseases.

\section{Aim of the Study}

The present study aims to identify mechanisms favoring healthy eating patterns that allow the management of daily food practices in face of a nutritionally challenging environment.

\section{Methods}

This exploratory qualitative study aimed to identify mechanisms favoring healthy eating behaviors in a Chilean adult population. Participants were recruited within a primary health care center in an urban municipality of the Metropolitan Region of Santiago, Chile. One hundred prospective participants were randomly selected from the database of a previous study [21] consisting of 400 adults between 35 and 65 years old without type 2 diabetes and without obesity. Those who were currently under pharmacological treatment with hypoglycemic agents and corticosteroids, consumed supplements or medications for weight control, presented pathologies that affect nutritional status, underwent bariatric surgery, presented eating disorders, and pregnant and nursing women were excluded.

Prospective participants who fulfilled the criteria were summoned to the health center for a nutritional evaluation. Trained nutrition students measured participants' weight and height to determine Body Mass Index (BMI) and obtain an updated nutritional status. A dietary assessment was performed though a 30-day food frequency questionnaire [22,23] and diets were classified according to the Healthy Eating Index (HEI) [24]. Participants with scores in the $75^{\text {th }}$ percentile were classified as healthy eaters and selected for interviews.

The final study population consisted of 16 people, 14 females and 2 males obtained HEI within the $75^{\text {th }}$ percentile and participated of the interviews. The average age of the group was 51 years, men being slightly older. $37.5 \%$ of participants had a normal nutritional status, while $62.5 \%$ were overweight. The socioeconomic level of this group was concentrated in the middle stratum. Half of the participants completed secondary education, while only $7.6 \%$ complete higher-level education. Characteristics of the participants are shown in table 1.

Semi-structured face-to-face interviews were conducted in Spanish, between October 2016 and January 2017 at respondents' workplace or homes; while others preferred to be interviewed at the health center. The interview guide addressed four dimensions of food: (i) individual, social and environmental factors influencing food choice; (ii) knowledge, beliefs, perceptions and attitudes regarding food; (iii) influential experiences in the development of dietary behaviors; (iv) mechanisms by which different factors guide dietary behaviors. The interviews were audio recorded and then 


\begin{tabular}{|l|l|l|l|}
\hline Characteristics & Total & Males & Females \\
\hline Age in years, average (SD) & $\begin{array}{l}51.6 \\
(6.64)\end{array}$ & $\begin{array}{l}54.5 \\
(9.19)\end{array}$ & $\begin{array}{l}51.2 \\
(6.55)\end{array}$ \\
\hline Nutritional status by BMI (\%) & & & \\
Normal & 37.5 & 0 & 100 \\
Overweight & 62.5 & 20 & 80 \\
\hline Socioeconomic status (\%) & & & \\
Upper class & 14.29 & 0 & 100 \\
Upper middle class & 50 & 14.29 & 85.71 \\
Middle class & 21.43 & 0 & 100 \\
Lower middle class & 14.29 & 0 & 100 \\
Lower class & 0 & 0 & 0 \\
\hline Educational level (\%) & & & \\
University, completed & 7.14 & 0 & 100 \\
Tech school, completed/UI & 21.43 & 0 & 100 \\
Tech school, incomplete & 0 & 0 & 0 \\
Secondary, completed & 50 & 14.29 & 85.71 \\
Secondary, incomplete & 7.14 & 0 & 100 \\
Primary, completed & 0 & 0 & 0 \\
Primary, incomplete & 14.29 & 0 & 100 \\
No education & 0 & 0 & 0 \\
\hline
\end{tabular}

Table 1: General characteristics of study participants. SD: Standard Deviation; BMI: Body Mass Index (kg/mt²); UI: University, Incomplete.

transcribed verbatim. Names of the participants were replaced by codes to protect their identity and confidentiality.

Interview transcripts were coded independently by two researchers through open, axial and selective coding. Codes were then discussed using the constant comparative method and based on Grounded Theory [25], comprehensive emerging themes were categorized upon consensus. CAQDAS Atlas.ti (Atlas.ti version 7.5. Scientific Software Development GmbH, Berlin, Germany, 2016) was used for interview transcript coding and analysis.

This study was reviewed and approved by the research ethics committee of the Faculty of Medicine of Universidad de los Andes, as well as the Western Metropolitan Health Service's Ethics Committee which oversees the primary health center addressed in this study. All participants gave written informed consent for the nutritional assessment and interviews.
When exploring the origins of healthy eating behaviors among adults, four themes emerged from the transcripts: 1) the physical and emotional roles of food; 2) food-related learning processes; 3) tools used to manage healthy diets and 4) health as the main mechanism that encourages healthy diets.

\section{Role and relevance of food}

Participants recognized food as important in the fulfilment of two roles. A first role related to the physical dimension of the body and health; and a second role related to the emotional dimension, concerning feelings generated and associated to foods and their emotional value.

Physical role: The impact of food. Participants identified an immediate repercussion of food on physical performance and well-being. Food is considered as a vital source of energy to carry out daily activities and needed to satisfying hunger, as depicted by participants P28 and P29. Furthermore, participants noted long-term, cumulative effects of eating habits on health, understanding food as a source of health or disease, as showed by participant P14.

- P28: ...Fundamental, feeding yourself is fundamental. If not, we wouldn't have energy, we would be sleepy, weak...

- P29: ... it gives me the energy that I need to work, to get through the day... your body is nourished and you can perform different activities, because if you do not eat you do not have energy. If you do not eat well you get sick, so in order for your body to be healthy and strong you have to eat, but healthy things.

- P14: I believe I'll have a better old age, since food has an influence on everything, even the skin, hair, mood... good food influences everything. [my husband and I] are now entering a different age and we must worry more about what we eat to have a good old age, if we make it to an older age.

Emotional role: Feeding and feelings: Participants expressed mainly positive feelings associated to food processes, as food generates a sensory pleasure and that is enjoyed while sharing with others. Whereas some others referred negative feelings, such as guilt, associated to the consumption of foods identified as unhealthy:

- Interv.: When you eat, does food give you any kind of feel-

\section{Results}


ings?

- P28: Feelings... sometimes guilt. I don't know, I'm greedy, I prefer sweet things rather than salads, so for example, I have cake, and then I say, "I shouldn't have eaten it, or I should have eaten half of that".

On the other hand, participants who mentioned having changed former eating habits and acquired healthier ones expressed a sense of emotional well-being generated by inner tranquility, feeling determined by a sense of coherence reinforced positively by what must be done and what is actually done in terms of diet.

- P25: I feel good when I eat... because I know I'm eating well...

- P14: I feel calmer because I know I'm trying [to eat healthy]. If it weren't for the sugar I eat, everything else would be fine, because I eat vegetables, fruits, a little bit of everything...

\section{The food learning triad}

A common learning process regarding food and eating behaviors emerged from participants' narratives. This food learning process is composed of three articulated stages that simultaneously precede and succeed each other over a continuous and dynamic process constructed by the integrating knowledge and experiences, for which it can undergo important modifications throughout life. The stages that comprise the Food Learning Triad entail a cognitive, reflective and modification in praxis stage, represented in figure.

Cognitive stage: At this stage, evidence-based information and theoretical knowledge obtained from different sources (e.g. provided by health professionals or from the media) provides the necessary input for participants to consider changing certain food practices. Thus, the cognitive stage emerges as the moment in which participants incorporate information into their mental schemas.

- P10: I used to eat a lot of fried foods, but now I'm eating healthier, lots of salad. They [nutritionists] taught me what salads were, what kind of vegetables they should have, how I had to eat them... all the food groups... That's what they taught me and I'm better.

Reflective stage: After receiving and incorporating information in the cognitive stage, a first evaluation is performed when applying said information into life experiences. The reflective stage, therefore, represents the visualization of possible benefits that could

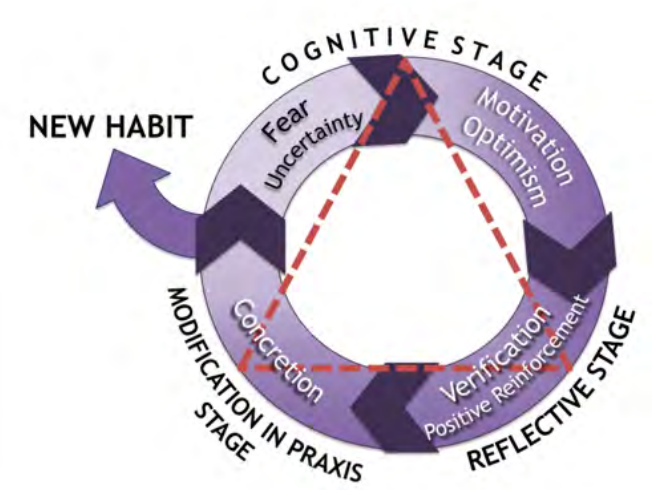

Figure : Conceptual model of the Food Learning Triad and its stages.

be achieved based on information acquired in the cognitive stage, versus the barriers and difficulties of its practice. At this stage, participants refer testing small behavioral changes to experience the expected benefits directly and determine whether they are able to cope with the challenges that arise during those changes. This is illustrated by the following extract:

- P25: What happened is that I didn't cook much before, because I got home late from work, I arrived tired to do chores and didn't really cook. I bought a lot of meals at work, grilled chicken and fries, a big portion, so I think that was bad for me because I was already overweight... And now we do these [healthy] meals, I feel really well. [...] I usually feel good now. All the meals we make, for example if it's chicken, I eat a bit of breast, and I feel good now, I feel my body is good, super good, I don't go around with a heavy body.

Modification in praxis stage: This stage entails an assessment process of the Reflective stage. The evaluation of the behavior is meaningful, that is, the perceived benefits have a positive value compared to the challenges faced during change, the adoption and preservation of the behavior occurs. On the other hand, when the assessment of encountered difficulties outweighs the experienced benefits following the new behavior, the possibility of change and its maintenance over time is hindered. This assessment process is illustrated by the following extract: 
- P10: I looked at myself in the mirror, nothing looked good, even if I wasn't so fat, but I still felt bad in clothes, in mood. But now I feel more like getting up, going to work. So that change has been good for me.

\section{Healthy eating tools}

Participants mentioned several tools they use to manage eating healthily and making adequate food choices. These tools were classified into three groups: knowledge, resources and skills and motivation.

Knowledge: Three types of food-related knowledge emerged from the transcripts. The first kind is factual knowledge, concerning different levels of food and nutrition knowledge and facts. On a first level, participants identify the existence of food groups associated to healthy diets, based on features such as origin, quality and nutritional value. These food groups are preferred over others with unhealthy characteristics.

- P8: I know chocolate is not bad, depending on how it is. Chocolate with $70 \%$ cocoa is not bad, but those cheap chocolates are sickening.

- P29: Well, for me, healthy things are those that don't have much sugar [...] For example, vegetables are healthy, unlike pasta. For me that isn't very healthy, one can eat them occasionally, but not regularly because they provide carbohydrates and that is transformed into sugar.

On a second level, participants recognized being aware of adequate portions, and the importance of serving sizes according to specific life-stage needs. They also refer concern and knowledge related to proper food preparation and storage processes, which in some cases is mentioned as harmful to health if not followed correctly.

All these levels of knowledge sum into the understanding of food having an influence on health, acting as protection from certain nutrition-related diseases or as a risk factor to acquire them. This is translated into self-knowledge and awareness of body signals generated throughout the life cycle as a response to eating habits:

- P8: At sixty (that's my age), one must be smart enough to realize... I know my body and I know what is good or bad for me...
The second type of food-related knowledge are myths and beliefs, which contrary to factual knowledge, are based on hearsay or participants' own perceptions. These myths and beliefs comprise ideas such as healthy diets having higher costs or obesity within families being caused by genetic hereditary factors (instead of acquired eating habits). The emerging myths and beliefs that stand out relate to specific negative or positive attributes assigned to food and their effects on health, such as sugar and carbohydrates being "poisonous" and farm/countryside products being wholesome foods. These myths and beliefs are exemplified by the following extracts:

- P8: They say that cow milk is bad ... They say that milk is bad, that Chinese don't drink milk so they don't have cancer...

- P14: I know that sugars are bad for the brain, I've done some research.

- P14: ...My mother sends eggs [from the south]. Those freerange eggs are healthier.

Resources and management skills: Two kinds of resources are identified by participants as relevant when dealing with food: time and money. However, participants mention that time and money are rather scarce and regardless they manage to use and distribute them efficiently to have healthy dietary behaviors. Participants recognize that, despite having a busy life, they are able to manage the time to perform healthy food processes, such as cooking; moreover, they do not perceive these practices as time consuming. Additionally, money is recurrently mentioned as a resource taken into consideration when making food choices, and budgets are carefully considered when purchasing food, as mentioned by one of the participants:

- P13: Time [for cooking] ... how long ... an hour, half an hour, I do not know, it's not much. I like cooking... But not cooking dishes that take a lot of time to elaborate; I have a simple and basic cuisine...

- P13: It depends on how much money I have. There are things that sometimes I can't buy. But if at a given moment I have money and I am going to buy food, meat goes last; I first look for vegetables, I can't go without bread, [non-caloric] sweetener, fruits, and milk.

Motivation: Motivation emerges as a necessary tool for conducting healthy eating behaviors. Participants mentioned internal and 
external incentives for eating healthily. On the one hand, internal motivation refers to the desire to take care of one's own diet for personal purposes, such as wanting to feel healthy, avoid illness and looking good physically:

- P23: At my 46 years of age, fortunately I don't have hypertension, I don't have diabetes, I don't have any chronic illness... I started to take care of myself particularly because I don't want to depend on medication at some point.

On the other hand, external motivation refers to the determination to take care of one's own diet to stay healthy because of others, particularly due to the responsibility of rearing children. Participants also refer external motivation when seeking to establish healthy food habits at home in favor of the health and well-being of other family members (children, grandchildren, partners).

- P17: They can prepare a barbecue full of sausages and no thanks, I'll pass. If I don't take care of myself, where will I end? I have six grandchildren and four daughters... So, I better [take care of diet], especially since I want to see my grandchildren with my children...

The food-health link: Mechanisms guiding healthy eating practices

Participants' narratives reveal certain mechanisms allowing them to face the daily challenges of eating healthily within obesogenic environments. These mechanisms comprise a deep realization of the importance of food for themselves and their families, identifying eating habits as health promoters, as well as causes of illness. Furthermore, participants mention being aware of the impact of food on their past and current health status, recognizing the link between food and health as the main mechanism that sustains their healthy diets. Specific mechanisms emerged from the interviews: Historic, Preventive, Reactive and Vicarious mechanisms.

Historic mechanism: Parent-influenced eating: Based on childhood memories and/or constructions generated from stories told by others (i.e. parents, siblings relatives, etc.), participants identified food patterns fostered by their parents as crucial influences on their current diet and health status. Some participants mentioned them as the reason they managed to grow healthily and remain healthful; while others refer to them as the cause of their current illness. Both cases are depicted by the following extracts:
- P17: I think that the food: beans, vegetables ... it was more nutritious, because we were satisfied. Did we get sick? ... We never got sick, we all turned out healthy.

- P18: You see; I feel that somehow the obesity I dragged during that time had something to do with the little [nutrition] education available for people in those days. I remember a chubby kid was considered a healthy kid.

Preventive mechanism: Conscious eating: Participants express awareness of the risks of unhealthy diets and the benefits of adequate eating habits for current and future health. The importance of this for an adequate ageing process is also taken into account. Such awareness drives them to be healthy eaters in order to avoid future diseases and have a healthier ageing process. A couple of participants illustrate:

- P14: "I know that I just have to eat well for my health, because I am older, so... my husband said " we are reaching the third age". That's why we have to take care of our health, for our old age".

- P23: "I mean, if I take care the risk decreases... Avoid getting diabetes one day, hypertension, I don't know if at some point, further down the line, in a few more years. But for now it's what I'm trying to do... avoid.

Reactive mechanism: Food for healing: In the presence of disease or its possible short term installation, the instrumental use of food as part of a health restoration process emerges as a mechanism. Participants mention how they confront health conditions and pathologies (overweight, fatty liver, hypertriglyceridemia, gastroesophageal reflux, etc.) with a dietary approach:

- P14: Now I am giving it [diet] more importance, because at my age... Before I ate a lot of junk food, not anymore. Now I make time, for example, lots of vegetables, fruits during the day, I try to eat more fish instead of meat, I try to make lots of vegetable tortillas. Since I had surgery a few months ago I'm taking care of my diet, I don't know why ... maybe to take care of myself so I don't get a new kidney stone.

- P10: I need surgery for an umbilical hernia that's bothering me. I looked into it years ago but stopped going to the hospital, I even lost the surgery appointment. Now I picked it up again, three months ago, they told me that if I wanted to get surgery I had to lose weight, and there everything started. 
Vicarious mechanism: All for one and one for all: Caring for children and/or family members' diet is manifested by participants as an altruistic interest to favor healthy food practices within the household. The family or social group's heathy diet is identified as a superior incentive that promotes developing and establishing dietary routines to attain and/or maintain good health for the whole group:

- P18: ...I try to pass good food practices on to my children. Therefore, in terms of being able to provide a healthy diet and teach them that, I consider myself paid... So when I spend the weekend cooking, I try to make everything balanced, try to give more importance to vegetables, try to add color to meals, try to make them entertaining so it will actually catch the children's attention. For example, a rich salad of lettuce, but with beans. Mix legumes, colors, vegetables... things that are healthy for them.

Participants also mention implementing family support systems for members who must face restrictive diets or change their eating habits due to specific health conditions. This special diet is (partially or completely) adopted by other family members even if they do not have the condition themselves, in order to provide a supportive food environment that allows a better adherence to the special diet. This is exemplified by one of the participants:

- P28: My dad has a kidney condition, so I'm super restricted [food wise]. On our refrigerator there is a list [this long] of what you can't eat, and [this short] of what you can eat. So, we try... my mom cooks almost with no salt and we try to avoid eating things that he can't eat so he doesn't see, or else he feels bad.

\section{Discussion and Conclusion}

This study sought to identify mechanisms that enable overcoming obesogenic environments amongst people that manage healthy eating habits, based on the Salutogenesis [8-11] perspective and through qualitative methods.

The study population was comprised only by people who obtained high scores in the Healthy Eating Index. Even though some of them were overweight, they had recently changed their eating habits and had adopted healthy diets. Most of the participants in this study (14 out of 16) were female, indicating that women are usually healthy eaters rather than men. These results coincide with national data [3]. Studies show that being female is a protective Salutogenic factor regarding food [13] and attribute the tendency of women having healthy diets to their strong beliefs in the importance of eating healthily [26].

Several resources used to accomplish healthy diets were identified. One of these resources is knowledge, comprised by formal science based nutrition knowledge provided by health professionals, as well as beliefs and myths acquired from popular sources. There is contradictory evidence regarding the impact of nutrition knowledge as an influential factor in healthy dietary behaviors $[4,27,28]$; however, food and nutrition knowledge is identified as one of the attributes of food literacy [29], thus it is important that health professionals generate nutrition educational measures to diminish the gap of formal knowledge regarding food and nutrition to facilitate patients making informed food decisions.

Research has identified time [27,30,31] and money [30] as main barriers for healthy eating. However, in the present study, healthy eaters recognized having the ability to optimize time and money to ensure a varied and sufficient diet of nutritional quality, despite acknowledging having limited free time and budgets due to their socioeconomic condition. Skills related to purchasing, preparing and handling food have also been identified as attributes of food literacy [29]. According to the Salutogenic Model, the ability to recognize and use resources (even though they are limited) for health promotion purposes is congruent to elements of Sense of Coherence (SOC) described by Antonovsky [8-10]. Studies have demonstrated an association between a high SOC and healthy dietary decisions [14-16]; therefore, the population interviewed may have this feature since they recognize having the ability to manage and prioritize time and budgets for healthy food despite limited availability of these resources. Having resource-management skills enables them to successfully face the challenge of healthy eating. This is associated to food literacy attributes of self-efficacy and confidence [29].

In this study, the relationship between food and health emerges as the main mechanism that promotes good dietary behaviors. Maintaining a healthy diet is done to benefit health either in a preventive or curative way, for themselves and their families. Healthy eaters from this study assign food a significant role in wellbeing. It is important for physical health as it is recognized as the source of nutrients and energy needed for everyday life, to prevent illnesses 
and reach old age in good conditions. At the same time, an emotional role is identified, associating the consumption of healthy foods to positive feelings, and unhealthy foods to negative feelings. Healthy eating interpreted as fundamental to reach a state of health and well-being has also been identified in other populations [28,32-34].

A food learning process was constructed and conceptualized, based on three correlated stages through which food-related learning takes place and favors performing and adopting healthy eating habits. This Food Learning Triad is a dynamic process that integrates knowledge, contemplation and experience that allow the modification and adoption of food behaviors. From a Salutogenic perspective, the Food Learning Triad is comparable with the three dimensions of SOC [8-10]. Firstly, the Triads' Cognitive Stage emerged as the moment when nutrition information is incorporated and taken into consideration for coping with food practices and environments. It is comparable to the SOC's comprehensibility dimension, its cognitive component which refers to the extent to which received information makes sense and the degree to which the challenge of coping with stressors is understood. Secondly, the Reflective Stage of the Triad in which an evaluation process of one's capability of performing certain behaviors takes place can be associated to the manageability dimension of SOC, its behavioral component referring to the extent to which resources needed to cope with stressors are perceived as accessible. During this stage, the emotional role of food identified in this study is materializes, as positive mood states arise when performed food behaviors and food goals are congruent, reaching feelings of achievement and tranquility; whereas negative feelings like guilt arise when behaviors do not meet established food goals. Finally, the Triad's Modification in Praxis Stage in which the new behavior is accepted as useful and beneficial for set food goals relates to the meaningfulness dimension, the motivational component of SOC that refers to the degree to which life makes sense and there is a desire to cope with stressors.

This study was the first of its kind in attempting to unravel the mechanisms by which Chilean adults overcome the obesogenic environment through a Salutogenic perspective and it is not without limitations. Firstly, the study population's homogeneity, being mostly women given by the sampling strategy used. Through intentional sampling, a greater male participation could have been obtained, gathering relevant data to detect more elements that facili- tate healthy eating in men. Secondly, conducting semi-structured interviews in various meetings would allow a better understanding of the topic investigated, as compared to a single semi-structured interview session; since it requires participants to recall life experiences, and reflect on the meaning of food and why it is important to them. Regardless of these limitations it was possible to explore how the value of health is the main drive for healthy food practices among healthy eaters.

\section{Implications for Research and Practice}

Further research exploring the mechanisms that allow successfully coping with nutritionally challenging food environments should focus on different age groups, socioeconomic status and ethnic origin. Additionally, special attention should be payed to healthy eating men, as well as people with NCDs who manage to adequately control their condition to gain greater scope of promoters of healthy food behaviors.

The health-food link identified in this study as the main mechanism that supports healthy eating habits may provide nutritionists and dietitians with a better understanding for approaching food behavior change by dealing with patient's perception of susceptibility to nutrition related diseases. Additionally, the Food Learning Triad may serve as a useful tool for nutrition education interventions, establishing a closer link between patients' motivations and interests and the nutritional needs detected.

\section{Bibliography}

1. Albala C., et al. "Nutrition transition in Chile: determinants and consequences". Public Health Nutrition 5.1 (2002): 123-128.

2. Vio F., et al. "Nutrition transition in Chile revisited: mid-term evaluation of obesity goals for the period $2000-2010$ ". Public Health Nutrition 11.4 (2008): 405-412.

3. Ministerio de Salud. Gobierno de Chile. Encuesta Nacional de Consumo Alimentario. [Nacional Food Consumption Survey] (2010).

4. Ministerio de Salud. "Gobierno de Chile. Encuesta Nacional de Salud. [Nacional Health Survey] (2010).

5. Malik VS., et al. "Global obesity: trends, risk factors and policy implications". Nature Reviews Endocrinology 9.1 (2013): 1327. 
6. Lemmens VEPP., et al. "A systematic review of the evidence regarding efficacy of obesity prevention interventions among adults". Obesity Reviews 9.5 (2008): 446-455.

7. Ministerio de Salud. Gobierno de Chile. Nutrición Políticas Públicas en Alimentación y Nutrición [Nutrition Public Policy in Food and Nutrition] (2017).

8. Antonovsky A. "The salutogenic model as a theory to guide health promotion". Health Promotion International 11.1 (1996): 11-18.

9. Lindström B and Eriksson M. "Salutogenesis". Journal of Epidemiology and Community Health 59.6 (2005): 440-442.

10. Lindström B and Eriksson M. “The Hitchhiker's Guide to Salutogenensis. Helsinki: Folkhälsan Research Centre, Health Promotion Research and the IUHPE GWG-SAL (2010).

11. Mittelmark MB., et al. "The Handbook of Salutogenesis". Springer (2017).

12. Swan E., et al. "Profiling healthy eaters. Determining factors that predict healthy eating practices among Dutch adults". Appetite 89 (2015): 122-130.

13. Sutherland S. "Directing food practices towards health from a Salutogenic perspective. Exploring food literacy, GRRs and life experiences in students leaving the nest [MSc]. Wageningen University (2014).

14. Packard Packard CJ., et al. "Interaction of personality traits with social deprivation in determining mental wellbeing and health behaviours". Journal of Public Health 34.4 (2012): 615624 .

15. Ahola AJ., et al. "Sense of coherence, food selection and leisure time physical activity in type 1 diabetes". Scandinavian Journal of Public Health 40.7 (2012): 621-628.

16. Lindmark U., et al. "Food selection associated with sense of coherence in adults". Nutrition Journal 4.1 (2005): 9.

17. Mattila ML., et al. "Sense of coherence and health behaviour in adolescence". Acta Paediatrica, International Journal of Paediatrics 100.12 (2011): 1590-1595.

18. Myrin B and Lagerström M. "Health behaviour and sense of coherence among pupils aged 14-15”. Scandinavian Journal of
Caring Sciences 20.3 (2006): 339-346.

19. Suraj S and Singh A. "Study of sense of coherence health promoting behaviour in north Indian students". Indian Journal of Medical Research 134.11 (2011): 645-652.

20. Ray C., et al. "The role of parents' sense of coherence in irregular meal pattern and food intake pattern of children aged 1011 in Finland". Journal of Epidemiology and Community Health 63.12 (2009): 1005-1009.

21. Urquidi C., et al. "Rendimiento y Adaptación del cuestionario FINDRISC para la pesquisa de diabetes mellitus tipo 2 y prediabetes en una población de atención primaria en Chile”.

22. Shim J-S., et al. "Dietary assessment methods in epidemiologic studies". Epidemiology and Health 36 (2014): e2014009.

23. Urteaga $C$ and Pinheiro AC. "Investigación alimentaria: consideraciones prácticas para mejorar la confiabilidad de los datos [Food intake research: Some practical considerations to improve reliability of the results]". Revista Chilena de Nutricion 30.3 (2003): 235-242.

24. Pinheiro AC and Atalah E. "Propuesta de una metodología de análisis de la calidad global de la alimentación [Proposal of a method to assess global quality of diet]". Revista Médica de Chile 133.2 (2005): 175-182.

25. Glaser BG and Strauss AL. "The discovery of grounded theory". Chicago: Aldine (1967).

26. Wardle J., et al. "Gender differences in food choice: The contribution of health beliefs and dieting". Annals of Behavioral Medicine 27.2 (2004): 107-116.

27. Williams LK., et al. "Optimising women's diets. An examination of factors that promote healthy eating and reduce the likelihood of unhealthy eating". Appetite 59.1 (2012): 41-46.

28. Petrovici D and Ritson C. "Factors influencing consumer dietary health preventative behaviours". BMC Public Health 6.1 (2006): 222.

29. Perry EA., et al. "Identifying attributes of food literacy: a scoping review". Public Health Nutrition (2017): 1-10.

30. Kearney JM and McElhone S. "Perceived barriers in trying to eat healthier - results of a pan-EU consumer attitudinal sur- 
vey". British Journal of Nutrition 81.1 (1999): S133-S137.

31. Welch N., et al. "Is the perception of time pressure a barrier to healthy eating and physical activity among women?" Public Health Nutrition 12.7 (2009): 888-895.

32. Bisogni CA., et al. "How People Interpret Healthy Eating: Contributions of Qualitative Research". The Journal of Nutrition Education and Behavior 44.4 (2012): 282-301.

33. Bouwman LI., et al. "I eat healthfully but I am not a freak. Consumers' everyday life perspective on healthful eating". Appetite 53.3 (2009): 390-398.

34. Sun YHC. "Health concern, food choice motives, and attitudes toward healthy eating: The mediating role of food choice motives". Appetite 51.1 (2008): 42-49.

\section{Assets from publication with us}

- Prompt Acknowledgement after receiving the article

- Thorough Double blinded peer review

- Rapid Publication

- Issue of Publication Certificate

- High visibility of your Published work

Website: www.actascientific.com/ Submit Article: www.actascientific.com/submission.php Email us: editor@actascientific.com

Contact us: +919182824667 